\title{
EUR-Lex: el sistema de información jurídica de la Unión Europea
}

\author{
José Antonio Domínguez Rojas
}

EUR-Lex, Office des Publications, Unión Europea (Luxemburgo)

\section{Resumen}

El presente artículo describe la tipología de documentos incluidos en la base de datos EUR-Lex. Se hace mención a los diferentes actos (legales, jurisprudenciales y administrativos) del sistema jurídico de la Unión Europea y a las diferentes posibilidades de búsqueda de la base de datos. Por último, se presenta la estructura y el contenido de la noticia documental que recoge los metadatos añadidos en el análisis de dichos actos.

Palabras clave: Internet. Derecho de la Unión Europea. Sistemas de búsqueda.

\section{Abstract}

This article describes the type of documents included in the database EUR-Lex. It refers to the different acts (legal, case law and administrative) of the European Union Law system and to the different search possibilities of the database. Finally, it presents the structure and content of documentary notices that contain metadata added after these acts are analysed.

Keywords: Internet. European Union law. Searching.

EUR-Lex es el sistema de información jurídica de referencia de la Unión Europea. La responsabilidad de su gestión corresponde a la Oficina de Publicaciones Oficiales de las Comunidades Europeas, organismo interinstitucional que tiene como base jurídica la Decisión 2000/459/CE, CECA, Euratom, decisión del Parlamento Europeo, del Consejo, de la Comisión, del Tribunal de Justicia, del Tribunal de Cuentas, del Comité Económico y Social y del Comité de las Regiones, de 20 de julio de 2000, relativa a la organización y al funcionamiento de la Oficina de Publicaciones Oficiales de las Comunidades Europeas (número CELEX 32000D0459).

El presente artículo describe en su apartado primero las características esenciales de EUR-Lex. En el segundo se hace una referencia al acceso al Diario Oficial de la Unión Europea (DOUE) a través del referido sistema de información. A continuación, en el tercer apartado, se repasa la tipología de documentos incluidos 
en la base de datos y se hace mención a los diferentes actos jurídicos (legales, jurisprudenciales y administrativos) por medio de los cuales se desarrolla el derecho de la Unión Europea. En el cuarto se hace referencia a las diferentes posibilidades de búsqueda y navegación que en la base de datos puede realizar actualmente el usuario. A continuación, en el quinto, se describe la estructura y el contenido de la noticia documental que recoge los metadatos añadidos en el análisis de dichos actos. Por último, en el apartado sexto se hace una breve mención a los posibles futuros desarrollos del sistema.

\section{Características esenciales de EUR-Lex}

EUR-Lex es el resultado de la fusión, producida en 2004, entre la base de datos CELEX y la antigua web EUR-Lex. Este sistema juega hoy un doble papel: es la principal vía de consulta del Diario Oficial de la Unión Europea y el punto de acceso a una base de datos compuesta por más de 400000 noticias documentales. Estas noticias son el resultado del análisis legal y documental de los actos publicados en el DOUE y en la Recopilación de la Jurisprudencia del Tribunal de Justicia de las Comunidades Europeas, y de otros actos que, sin haber sido objeto de publicación oficial en los citados periódicos, son remitidos a la Oficina de Publicaciones por las distintas instituciones europeas para su difusión on-line.

El acceso a EUR-Lex es gratuito y de entre sus características principales podríamos destacar su inmediatez y su multilingüismo. La disposición de los documentos es prácticamente inmediata: los documentos se cargan en el mismo momento de su publicación en el DOUE o incluso antes, como ocurre con los de tipo jurisprudencial. En este caso, los textos provisionales de las sentencias dictadas por el Tribunal de Justicia de las Comunidades Europeas están a disposición de los usuarios de EUR-Lex minutos después de haber sido pronunciadas, sin esperar a su publicación en la Recopilación de la Jurisprudencia del Tribunal de Justicia. Los textos no publicados (como es el caso de los documentos COM de la Comisión) son incluidos en EUR-Lex por medio de una cadena automatizada de alimentación horas después de haberse recibido del organismo responsable de su publicación (en el caso citado de los documentos COM, la Secretaría General de la Comisión).

Pero sin duda uno de los puntos fuertes de EUR-Lex es el multilingüismo. El Diario Oficial es el único periódico de la UE publicado en todas las lenguas oficiales de la Unión. EUR-Lex, por tanto, debe reflejar esa heterogeneidad lingüística. A día de hoy, y salvo algunas excepciones relativas a las lenguas últimamente incorporadas al elenco de las oficiales (búlgaro, rumano e irlandés), todas las pantallas de EUR-Lex, así como los metadatos de las noticias documentales, están disponibles en todas las lenguas oficiales. En todo momento el usuario puede cambiar de lengua de trabajo (sin tener que volver al inicio de la búsqueda).

Scire. $15: 1$ (en.-jun. 2009) 15-32. ISSN 1135-3716. 


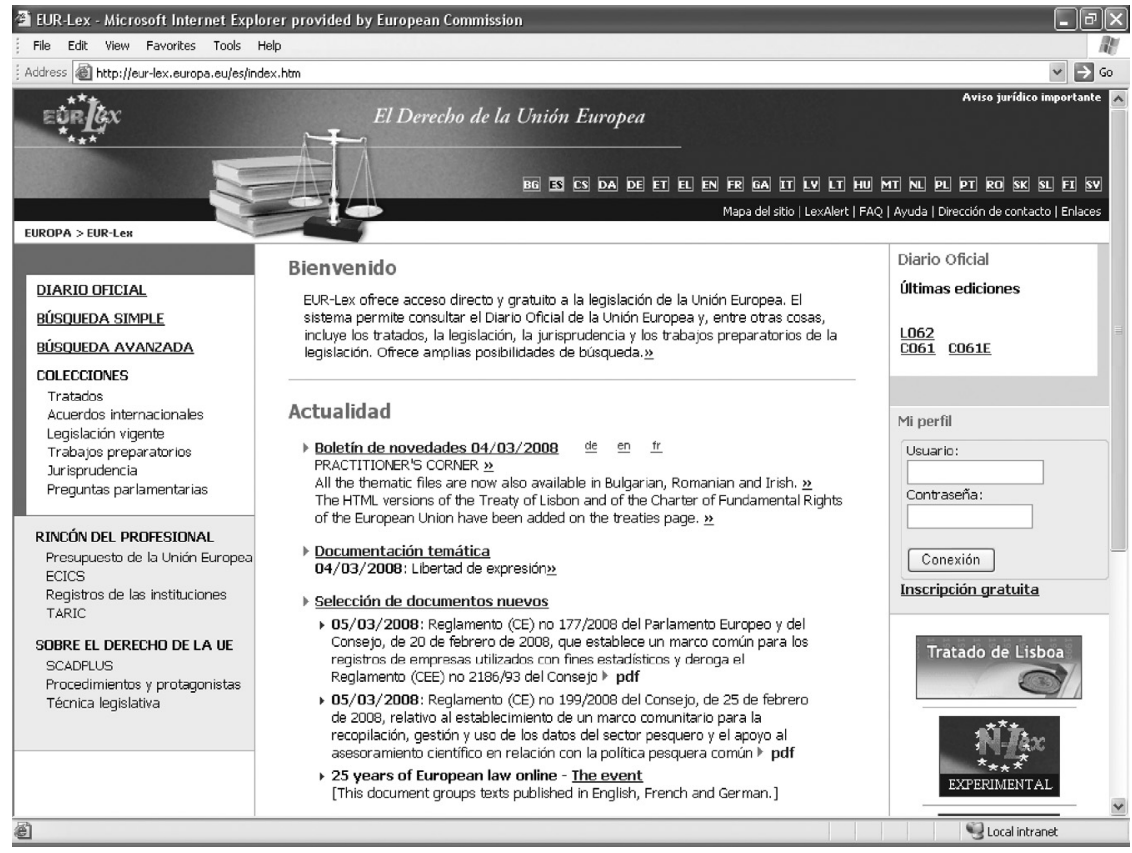

Figura 1. Página de inicio de EUR-Lex.

Para los textos de los actos en sí mismos, la realidad es distinta: lógicamente, y aunque con ocasión de cada ampliación son traducidos a las nuevas lenguas oficiales los actos que forman parte del acervo comunitario, hay una gran cantidad de actos que no constituyen legislación en vigor en el momento de la ampliación y que no son, por tanto, traducidos. Este aspecto lingüístico, como se verá más adelante, es importante a la hora de realizar las búsquedas, ya que todos los textos, en los diferentes idiomas, son indexados para facilitar la recuperación de la información por medio de términos.

\section{El acceso al Diario Oficial de la Unión Europea}

EUR-Lex es, primeramente, la principal fuente para acceder al Diario Oficial de la Unión Europea en formato electrónico. EUR-Lex permite consultar los actos publicados en las dos series del DOUE: L ("Legislación”) y C ("Comunicaciones e informaciones”). Tras su publicación, el Diario está en línea de manera prácticamente inmediata.

La obligatoriedad de la publicación de los actos de la Unión Europea (UE) viene fijada en el artículo 254 del Tratado constitutivo de la Comunidad Europea

Scire. $15: 1$ (en.-jun. 2009) 15-32. ISSN 1135-3716. 
(TCE), que habla de la necesaria divulgación en el DOUE de los reglamentos y las directivas que tengan como destinatarios a todos los Estados miembros. No obstante, diariamente muchos otros actos cuya publicación no es obligatoria aparecen en el Diario, siguiendo un deseo de transparencia e información al ciudadano.

No hace falta decir que, con el desarrollo de las tecnologías de la información y la comunicación, las suscripciones al Diario Oficial "papel" ceden cada día en favor de la consulta on-line. No obstante, debe recordarse que en el ámbito comunitario la versión auténtica del DOUE es, todavía, la impresa. La línea adoptada por algunos Estados miembros en el sentido de dotar de autenticidad a las versiones electrónicas de sus respectivas gacetas oficiales puede marcar el camino también en el ámbito comunitario.

Debe mencionarse no obstante, el suplemento $\mathrm{E}$ (electrónico) a la serie $\mathrm{C}$ del Diario: este solo existe en versión electrónica, sin soporte en papel, aunque en él se recogen actos cuya publicación no es obligatoria, generalmente emitidos por las instituciones en el curso del procedimiento legislativo (antes de su adopción formal), como posiciones comunes del Consejo o resoluciones legislativas del Parlamento en los ámbitos en los que actúa como colegislador, o preguntas y respuestas parlamentarias.

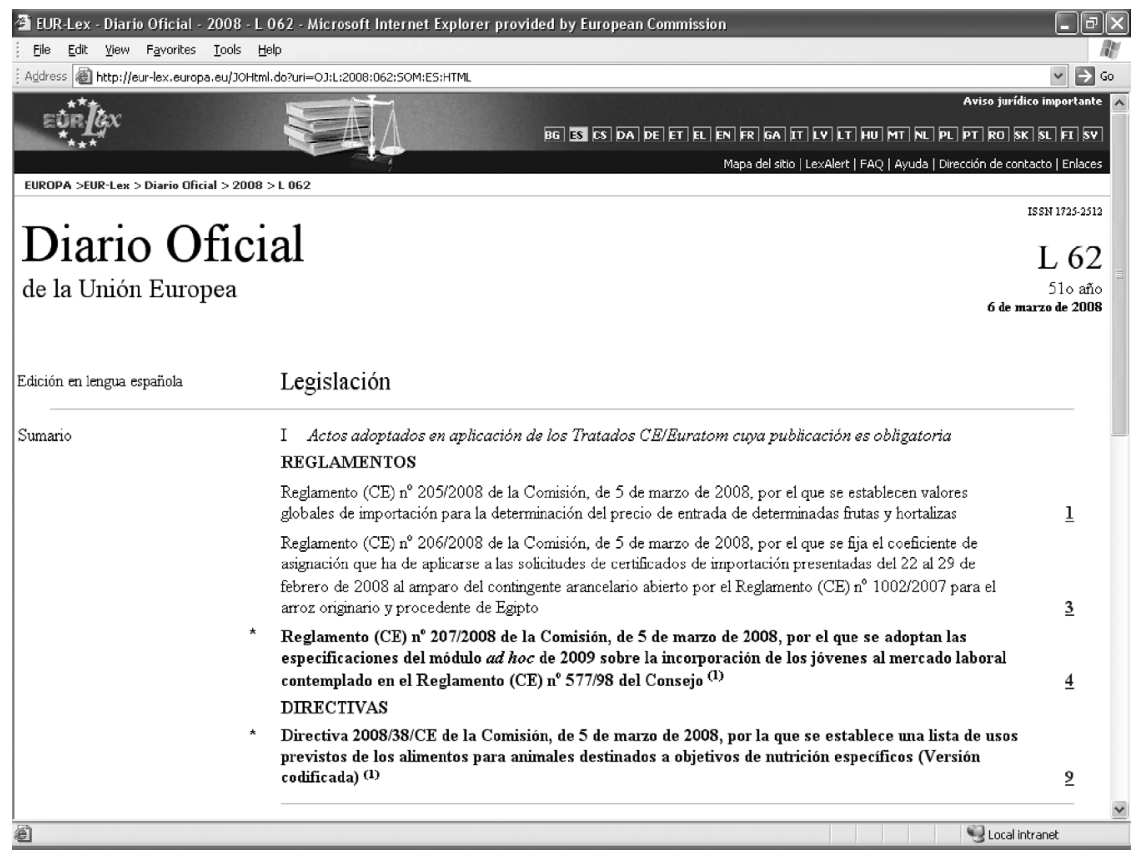

Figura 2. El DOUE en EUR-Lex: el número de página da acceso al documento PDF. 


\section{El contenido de la base de datos EUR-Lex}

Pero EUR-Lex no es solo un medio para consultar el Diario Oficial de la UE. Constituye igualmente una base de datos que sirve para acceder a más de 400000 noticias documentales compuestas por los textos de los actos adoptados y los metadatos, valor añadido resultado del análisis legal y documental de esos actos. En las líneas que siguen, mencionaré el contenido de la base, es decir, qué documentos pueden ser recuperados, haré una referencia a las estrategias de búsqueda, y finalmente me detendré en la presentación de resultados y en el contenido (los metadatos) de las noticias.

Los documentos alojados en EUR-Lex están organizados con arreglo a 11 sectores. Haremos una breve referencia a cada uno de ellos. Los sectores se identifican con el primer carácter del número CELEX, que es el identificador de las noticias. Este número se ha convertido en una referencia en la legislación comunitaria y se construye a partir de la combinación de un identificador del sector del acto, el año de su publicación, otro identificador sobre el tipo de documento y un número secuencial que puede estar ligado bien al recibido por el documento en su publicación en el DOUE, bien a la fecha de publicación. Así, por ejemplo, el 32008R0001 sería el Reglamento número 1 de 2008, mientras que el 22008A0124(02) sería el segundo acuerdo internacional publicado el 24 de enero de 2008.

\subsection{Sector 1: tratados}

En el sector 1 se incluyen los tratados fundacionales de las Comunidades y de la Unión Europea, sus tratados modificativos, los sucesivos tratados de adhesión y tratados específicos, tal vez menos conocidos (Tratado de Fusión de los Ejecutivos de las Comunidades Europeas de 1965, tratados modificatorios de determinadas provisiones presupuestarias y financieras de 1970 y 1975, tratado que modifica el estatuto del Banco Europeo de Inversiones, de 1975, tratado sobre Groenlandia de 1985).

En este mismo sector pueden encontrarse actos que no han llegado a tener vigencia, como el Tratado Constitucional, o actos que (en el momento de redactar estas líneas) no están todavía en vigor pero que han sido publicados en el DOUE para información, como el Tratado de Lisboa. Igualmente, las versiones consolidadas de los tratados originarios que el Consejo de la UE va produciendo tras cada tratado modificador o cada ampliación y que son publicadas en el Diario (en la serie $\mathrm{C}$, ya que no estamos en presencia de legislación, sino de instrumentos de información) son incluidas en este sector. Dada la complejidad de los actos legislativos comunitarios y, en particular, del derecho primario, son estas versiones consolidadas de los tratados las que resultan más consultadas por los usuarios.

La particularidad de las noticias documentales agrupadas en el sector 1 se encuentra en que, contrariamente a la regla general para el resto de los sectores, que

Scire. $15: 1$ (en.-jun. 2009) 15-32. ISSN 1135-3716. 
consiste en que por cada acto publicado existe una noticia documental multilingüe con un identificador (número CELEX) único (por ejemplo, un reglamento o una directiva genera una noticia), en este caso, y dada la importancia de los documentos aquí recogidos, se pretende realizar un análisis más exhaustivo, por lo que cada acto jurídico se divide en artículos, anexos, protocolos, declaraciones, actas finales, etcétera, y se genera una noticia multilingüe a partir de esas entidades. A cada una de esas noticias se le asocian los documentos primarios en sí mismos (los textos), generalmente en formato HTML o PDF.

Por poner unos ejemplos, el número CELEX 12006E001 identifica el artículo 1 (001) de la versión consolidada de 2006 (2006) del Tratado Constitutivo de la Comunidad Europea (E); por su parte, el CELEX 11992M/AFI/DCL/24 corresponde a la Declaración 24 (DCL/24) incluida en el Acta Final (AFI) del Tratado sobre la Unión Europea (M) de 1992.

\subsection{Sector 2: acuerdos internacionales}

En el sector 2 se incluyen los acuerdos firmados entre una o varias de las Comunidades Europeas, en solitario o junto con los Estados miembros (en este caso, denominados acuerdos mixtos), de un lado, y, de otro, terceros Estados y/u organizaciones internacionales. Todos los documentos generados en la actividad internacional de las Comunidades (piénsese en políticas de competencia exclusiva, o cuasi, de estas, como comercio o pesca) son alojados en este sector.

Además de los acuerdos propiamente dichos, en este sector se incluyen los actos de órganos (administrativos o parlamentarios) creados por dichos acuerdos, órganos tan prolíficos en la producción de documentos como el Comité Mixto del Acuerdo sobre el Espacio Económico Europeo o la Asamblea Parlamentaria prevista por el Acuerdo de Cotonú entre la Comunidad y los Estados ACP.

\subsection{Sector 3: legislación}

Aquí se recogen los bien conocidos reglamentos, directivas, recomendaciones y dictámenes de los órganos comunitarios, pero también otros actos menos populares y que forman parte igualmente del derecho derivado: actos de la política exterior de seguridad y defensa y de la cooperación policial y judicial en materia penal (no comunitarios, como es sabido, sino de la Unión Europea), líneas directrices del Banco Central Europeo, resoluciones y declaraciones.

\subsection{Sector 4: legislación complementaria}

En este sector están incluidos los acuerdos entre Estados miembros y las decisiones de los representantes de dichos Estados, actos de difícil definición formal y ante los que hay que adoptar un enfoque pragmático. No estamos aquí por tanto en presencia de actos producidos por las instituciones europeas (Consejo, Parlamento, Comisión), sino por los mismos Estados miembros, en ocasiones en ma- 
terias en las que los propios tratados lo han previsto así. Actos como las conclusiones de los Estados miembros reunidos en el seno del Consejo para establecer líneas directrices de futuras políticas o acuerdos relativos a cooperación en materia de ejecución de sentencias serían ejemplos de noticias incluidas en este sector.

\subsection{Sector 5: actos preparatorios}

En este sector se incluyen los actos adoptados en el curso del procedimiento legislativo. Generalmente el expediente legislativo empieza con una propuesta de la Comisión (mediante los llamados documentos COM), que sirve para poner en marcha el mecanismo, emplazando a los diferentes actores del proceso a que se posicionen por medio de los actos adecuados y según el procedimiento legislativo que corresponda según lo establecido por los tratados.

Además de la propuesta de la Comisión (o la iniciativa de los Estados miembros en ciertas materias), se incluyen en este sector las posiciones comunes del Consejo, las resoluciones legislativas del Parlamento, las opiniones del Comité Económico y Social Europeo y del Comité de las Regiones, y los dictámenes del Banco Central Europeo y el Tribunal de Cuentas Europeo.

\subsection{Sector 6: jurisprudencia}

Se incluyen en este sector los documentos generados por actividad judicial de las Comunidades Europeas: sentencias, autos, conclusiones y dictámenes del Tribunal de Justicia, del Tribunal de Primera Instancia, del Tribunal de la Función Pública y de los abogados generales.

\subsection{Sector 7: medidas nacionales de ejecución}

El sector 7 recoge los actos jurídicos adoptados por los Estados miembros para la ejecución de las directivas. Como es bien sabido, las directivas (art. 249 TCE) obligan al Estado miembro destinatario en cuanto al resultado que deba conseguirse, dejando a las autoridades nacionales la elección de la forma y los medios. En este sector, EUR-Lex recoge, pues, no derecho comunitario, sino derecho interno, es decir, actos adoptados por las autoridades legislativas o administrativas de los Estados miembros.

En este momento, el sector 7 de EUR-Lex no muestra los textos de las medidas nacionales de ejecución dictadas por las autoridades nacionales, sino solo una referencia a sus títulos y a los diarios oficiales nacionales en los que se han publicado. Es una buena forma, sin embargo, de tener una visión global sobre el cumplimiento que a una determinada directiva han dado los diversos Estados miembros.

Si se realiza, por ejemplo, una búsqueda por medio del número CELEX 72004L109, se obtendrá la lista de disposiciones nacionales que los diferentes Estados miembros han comunicado a la Comisión Europea como prueba de la correcta transposición a su derecho interno de la Directiva 109 de 2004.

Scire. $15: 1$ (en.-jun. 2009) 15-32. ISSN 1135-3716. 


\subsection{Sector 9: preguntas parlamentarias}

Las preguntas dirigidas por los parlamentarios europeos a la Comisión y al Consejo se cargan en el sector 9 de EUR-Lex tras la publicación en el Diario Oficial de la respuesta de la institución a la que van dirigidas. Estas preguntas pueden ser formuladas con petición de respuesta escrita y publicadas en el DOUE (preguntas escritas); abordadas en las sesiones parlamentarias y publicadas en los debates del Parlamento Europeo (preguntas orales); o planteadas en los momentos reservados para ello en cada período de sesión (preguntas formuladas en el turno de preguntas).

\subsection{Sector 0: legislación consolidada}

La Oficina de Publicaciones tiene entre sus responsabilidades la de proveer de versiones consolidadas de la legislación. En EUR-Lex, por tanto, se ponen en línea documentos PDF donde se integra la versión inicial del acto legislativo con las modificaciones que dicho acto ha sufrido. Estos actos no tienen valor legal, sino que sirven solamente como herramienta de documentación. No obstante, como es lógico, cuando el usuario habitual se enfrenta a un reglamento o una directiva que

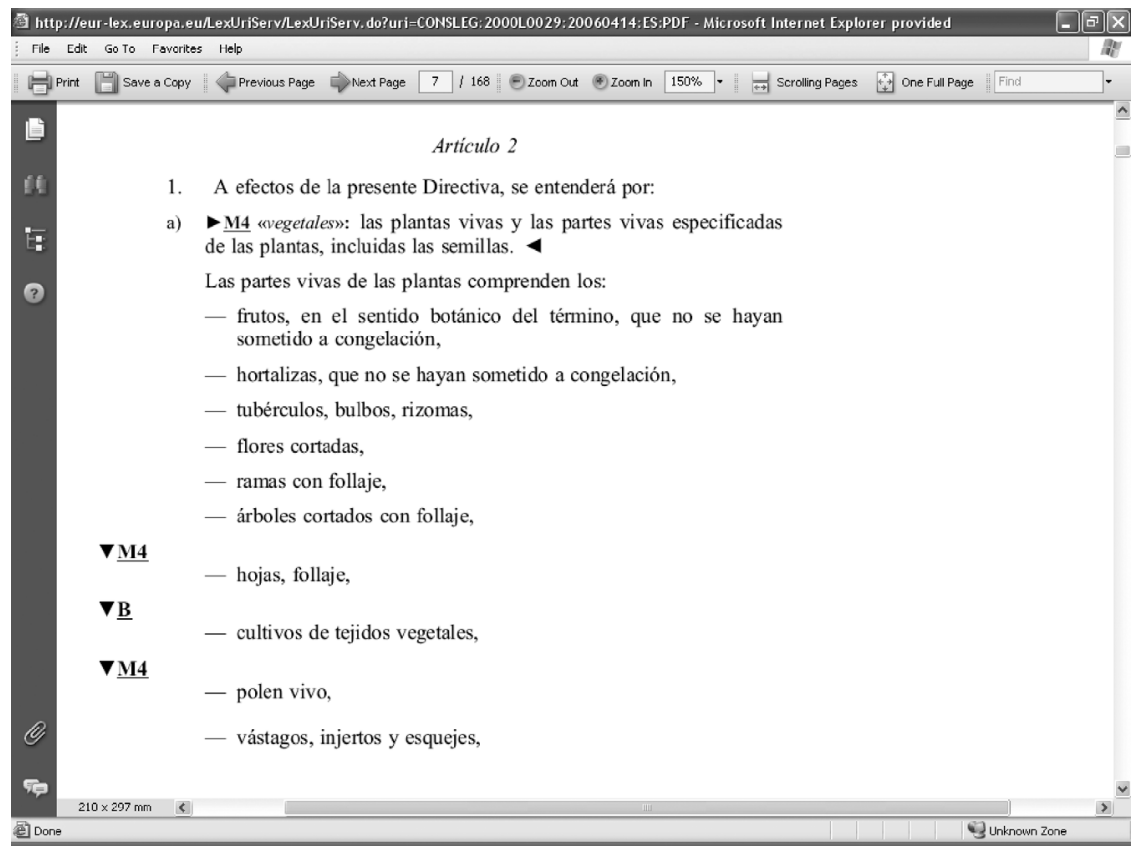

Figura 3. El PDF de un texto consolidado: el usuario encuentra el texto básico (B) y las modificaciones (M1, M2, etcétera). 
han sido modificados, en ocasiones, decenas de veces, acude normalmente a la última versión consolidada del acto.

\subsection{Sector C}

Hay una gran cantidad de documentos publicados para información en la serie $\mathrm{C}$ del Diario Oficial que no son analizados. Solo portan unos metadatos básicos y están dotados de un identificador, "C", ligado directamente a su referencia de publicación.

\subsection{Sector $\mathbf{E}$}

Por su incidencia en la legislación comunitaria, se decidió incluir en EUR-Lex, y analizar, los actos publicados en el DOUE procedentes de la Asociación Europea de Libre Comercio. Los actos, por tanto, de la Autoridad de Vigilancia de la EFTA (sus siglas en inglés), de su Comité Permanente y de su Tribunal, una vez publicados, son incluidos aquí.

\section{Las posibilidades de búsqueda en EUR-Lex}

Toda la información incluida en EUR-Lex resulta accesible a través de dos vías: la búsqueda simple y la avanzada. Además, EUR-Lex ofrece varias posibilidades de navegación.

\subsection{Búsqueda simple}

El usuario puede, utilizando la pantalla de búsqueda simple, recuperar documentos a través de palabras o expresiones en el título o en el texto del documento, combinando varias expresiones o excluyendo otras tantas de la búsqueda; igualmente, la recuperación de las noticias es factible a través de las fechas de adopción, publicación, entrada en vigor y fin de validez de los distintos actos jurídicos, con la posibilidad de elegir entre fechas concretas o periodos; también es posible realizar búsquedas utilizando el repertorio de la legislación comunitaria en vigor o recuperando las noticias que han sido indexadas con un específico descriptor del tesauro Eurovoc.

Otras opciones de búsqueda de la información se refieren a los identificadores de las noticias: un documento puede ser recuperado a través de su referencia (o número natural, es decir, el número atribuido al acto en el momento de hacerse público, como puede ser, por ejemplo, el Reglamento 110 de 2006 o el documento COM de la Comisión número 50 de 2007), a través de su número CELEX (que, como ya se ha apuntado es un identificador atribuido a todos los documentos de la base, salvo a aquellos incluidos en el sector $\mathrm{C}$, y que es el resultado de un análisis humano - no automatizado - del documento), o directamente a través de las versiones consolidadas de los textos legislativos, por medio del recurso a la navegación en el sector 0 . 


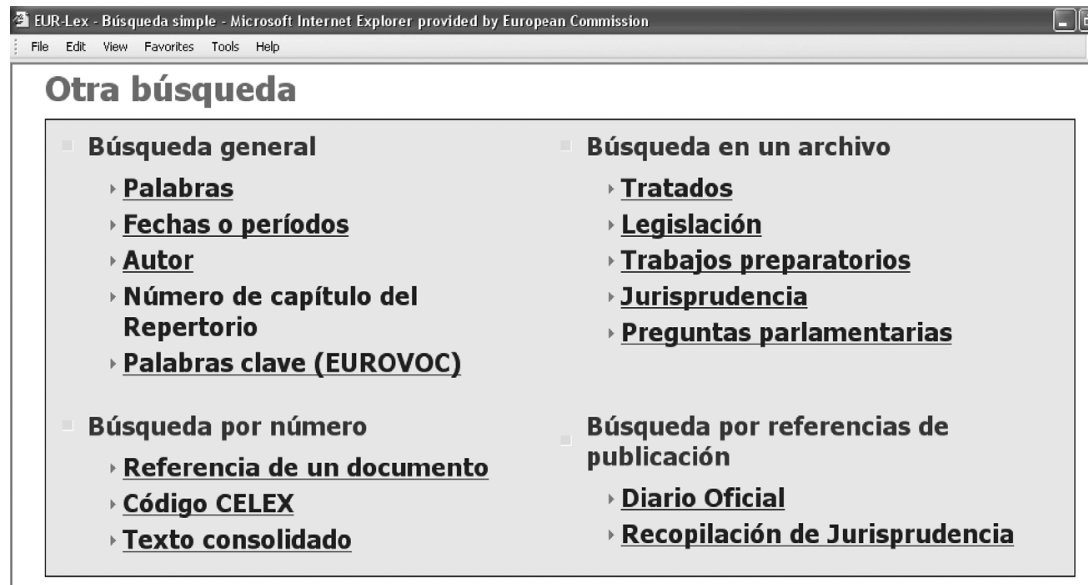

\section{Búsquedas anteriores}

- Historial de la búsqueda

- Búsquedas guardadas

- Mis expedientes

Figura 4. Pantalla principal de búsqueda simple.

El usuario también tiene la opción de buscar la información por tipo de documento: tratados, legislación, actos preparatorios, jurisprudencia o preguntas parlamentarias. Además de ello, cuenta con la posibilidad de recuperar el documento buscado a través de la referencia de publicación del acto en el Diario Oficial o en la Recopilación de la Jurisprudencia del Tribunal de Justicia.

Por último, la búsqueda simple ofrece la opción de recuperar y combinar búsquedas ya realizadas durante la sesión de consulta.

\subsection{Búsqueda avanzada}

La búsqueda avanzada es una herramienta para cuyo uso es necesario un cierto dominio de la base de datos, así como determinados conocimientos jurídicos. $\mathrm{Al}$ contrario de lo que sucedía con la búsqueda simple, aquí todos los campos son interrogables, y la información se recupera por medio de descriptores que están alojados en una serie de tablas de traducción (no olvidemos que podemos estar trabajando en cualquiera de las 23 lenguas oficiales).

Podemos interrogar al sistema mediante queries simples como, por ejemplo, Código_CELEX $=32005 \mathrm{R}+$ AND Título $=$ arroz 
que nos servirá para recuperar todos los reglamentos publicados en 2005 en cuyo título aparezca la palabra arroz, pero también llegar a queries más complejas, como

(Fundamento_jurídico $=(12002 \mathrm{E}+$ OR 12002M+) AND Fecha_del_documento $>=$ 2007/01/01) OR (Actos_citados) $=(12002 \mathrm{E}+$ OR 12002M+)

AND Fecha_del_documento $>=2007 / 01 / 01$ )

que nos permitirá recuperar todos los documentos cuya base legal sea, o en los que se citen, cualesquiera de los artículos de las versiones consolidadas en 2002 de los tratados de la Comunidad Europea o de la Unión Europea y cuya fecha de adopción sea igual o posterior al primero de enero de 2007.

La búsqueda avanzada de EUR-Lex permite guardar las búsquedas incluso después de haber cerrado la sesión y mostrar las noticias encontradas con arreglo a criterios más flexibles (el usuario elige los campos que quiere que le sean mostrados), y hace posible igualmente la extracción de datos de la base, que es remitida por el sistema por correo electrónico.

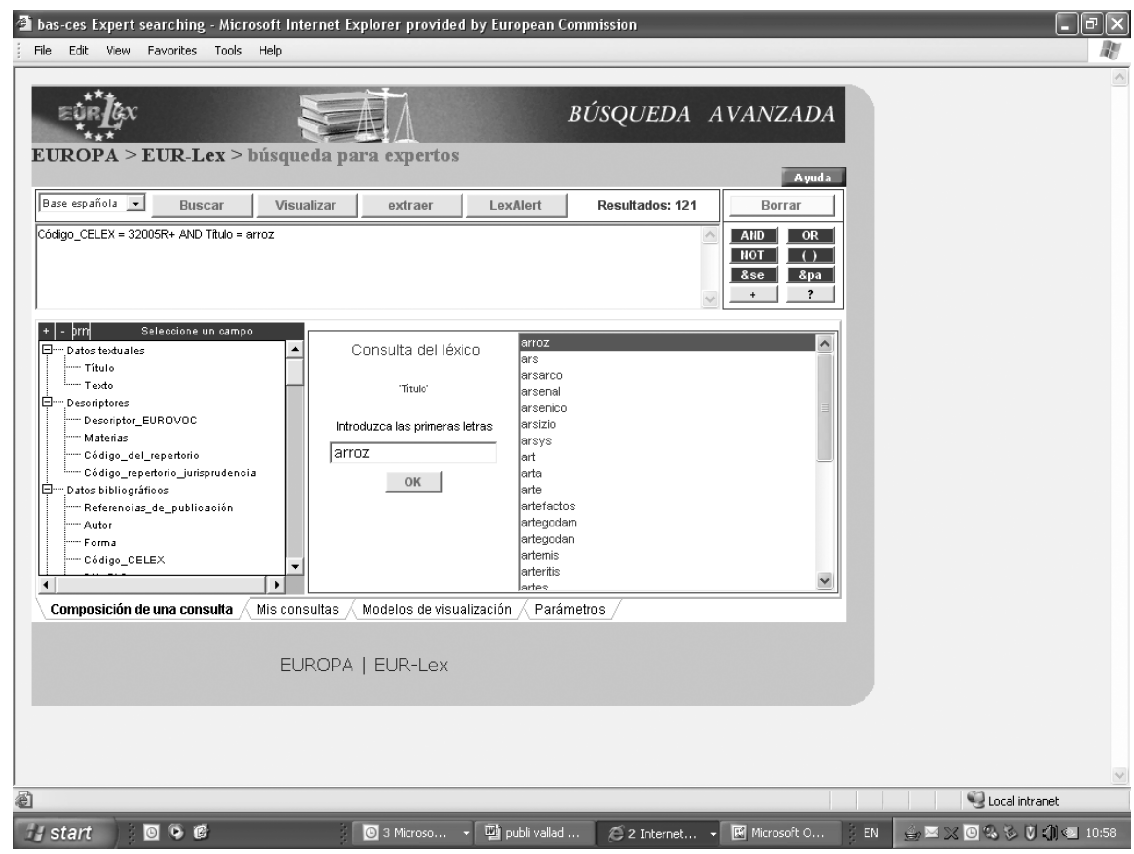

Figura 5. Búsqueda avanzada. 


\subsection{Navegación}

EUR-Lex permite igualmente la navegación en las diferentes colecciones de documentos incluidas en la base. Se facilita así la recuperación de la información de una manera intuitiva, sin necesidad de recurrir a interfaces de búsqueda, algunas veces percibidas (incluso la simple) como excesivamente complejas por los usuarios. El usuario debería poder llegar a recuperar los documentos deseados a través de la navegación en los tratados, los acuerdos internacionales, la legislación, los trabajos preparatorios en el proceso legislativo, la jurisprudencia y las preguntas parlamentarias.

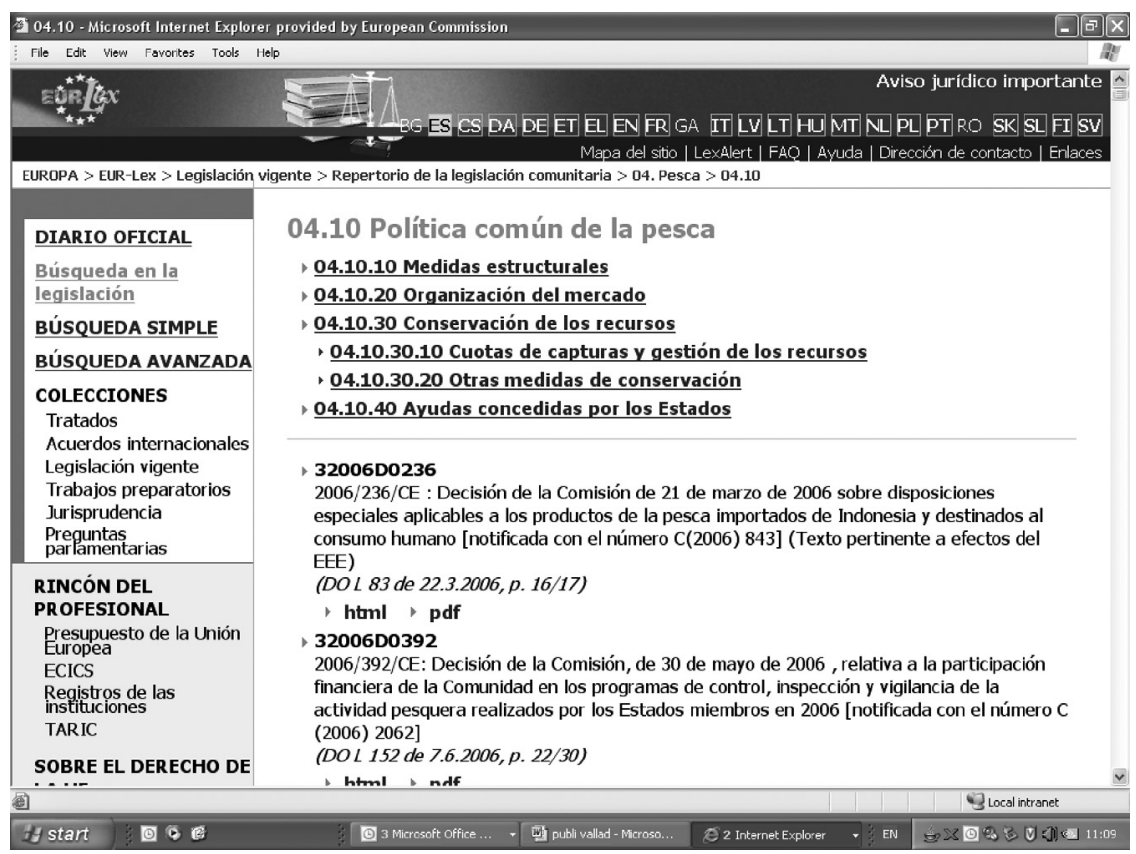

Figura 6. Navegación por colecciones.

\section{Resultados y noticia documental}

\subsection{Presentación de resultados}

Consecuencia de la búsqueda simple o de la navegación, EUR-Lex muestra una lista de resultados que por defecto son ordenados en orden cronológico inverso y en la que por cada acto se muestra el número CELEX, el título, la referencia de publicación y los formatos en los que pueden ser descargados los documentos. 


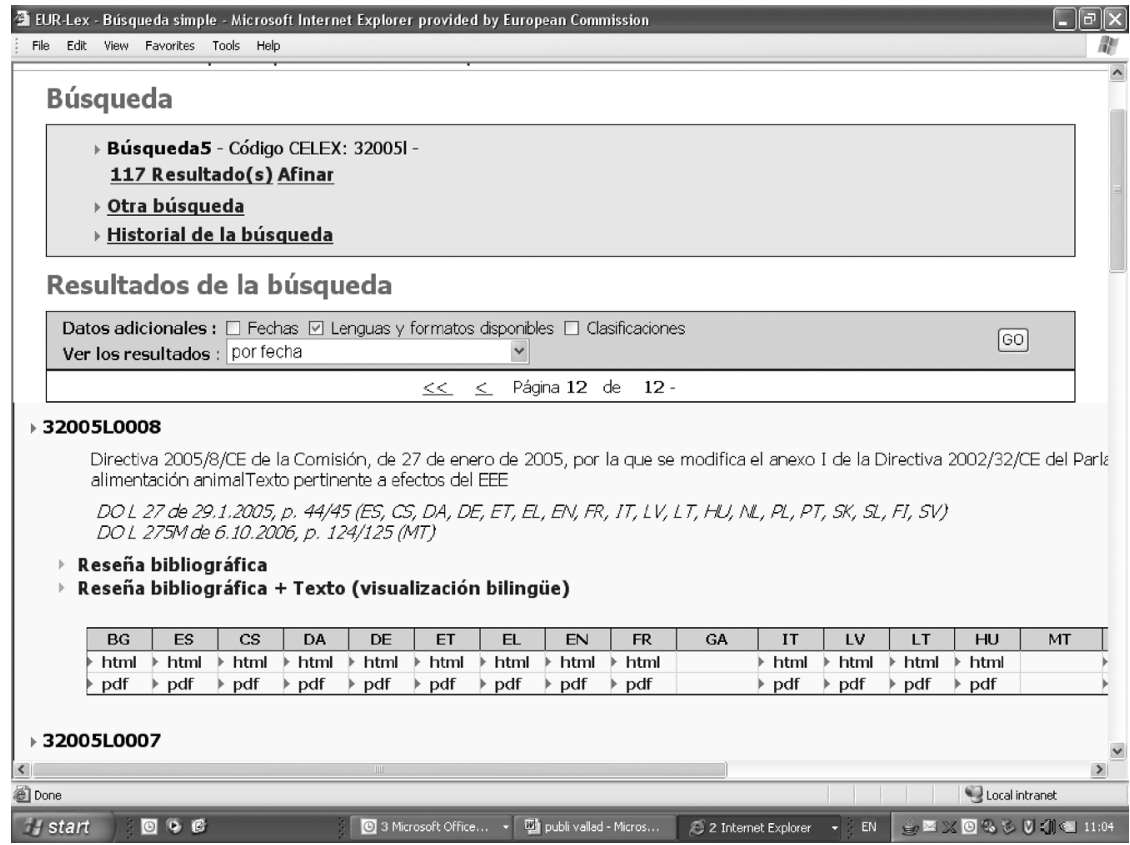

Figura 7. Navegación por colecciones.

En este sentido, debe apuntarse que la mayor parte de los documentos de EURLex se encuentran en formato HTML, concretamente la legislación publicada en el DOUE desde principios de los años setenta; los actos jurídicos incluidos en las ediciones especiales en las nuevas lenguas oficiales correspondientes a las ampliaciones de la Comunidad de los años 1973, 1981, 1986, 1995, 2004 y 2007; la práctica totalidad de la jurisprudencia del Tribunal de Justicia y del Tribunal de Primera Instancia; los textos de las preguntas parlamentarias a partir de 1994; los documentos de la Comisión de las series COM y SEC desde 1999, y la colección de documentos merger (tipo de documento particular indexado con los números CELEX 3M y 3J, relativo a no oposiciones a operaciones de concentraciones de empresas o de empresas conjuntas — joint ventures_-) desde 1995.

EUR-Lex también ofrece a los usuarios la posibilidad de descargar los archivos en PDF. En particular, desde que el Diario Oficial, en 1998, se publica en este formato, todos los actos que provienen de esa fuente pueden ser recuperados de este modo. Hay más documentos que se encuentran en este formato, como los COM o los textos consolidados de la legislación. Algunos se encuentran además en MS Word (por ejemplo, los documentos COM desde 1999 o los proyectos de codificación de la Comisión desde 2007).

Scire. $15: 1$ (en.-jun. 2009) 15-32. ISSN 1135-3716. 


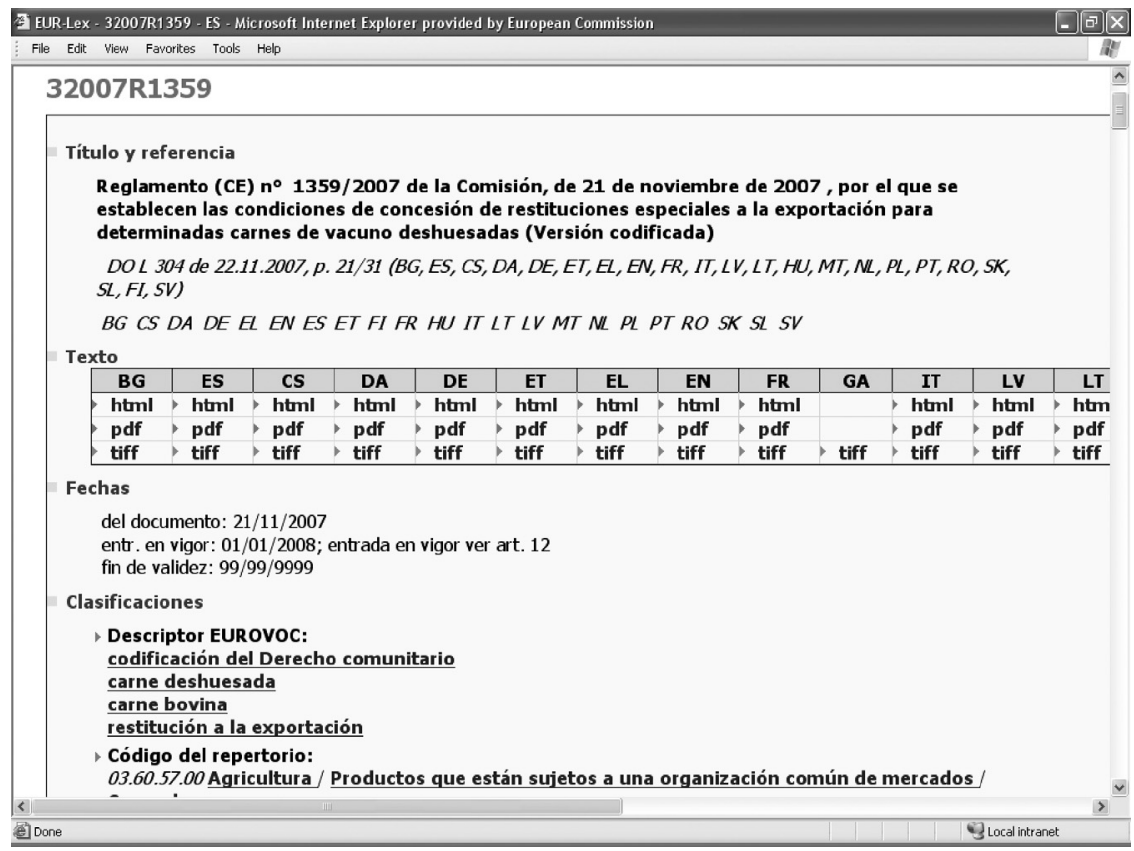

Figura 8. Noticia documental (I).

Por último, es posible, en la mayoría de los casos, obtener el documento deseado en formato TIFF (PDF-imagen), solicitándolo del archivo electrónico de la Oficina de Publicaciones a través de la noticia documental relativa al acto en cuestión. El documento será enviado a la dirección de correo electrónico indicada por el usuario.

\subsection{Noticia documental}

Haremos a continuación una breve referencia al contenido de la noticia documental, la cual se estructura en campos y grupos de campos que recogen, en cada una de las lenguas oficiales, toda la información jurídica y documental, valor añadido por el equipo de analistas a los actos publicados.

Con este propósito, hemos elegido como ejemplo el acto identificado con el número CELEX 32007R01359 (Reglamento de la Comisión número 1359 de 2007, perteneciente al sector 3, es decir, legislación). No todos los campos de que dispone EUR-Lex serán comentados; se ha elegido un ejemplo en el que se pueden encontrar los más comúnmente utilizados de acuerdo con la metodología de análisis.

La primera información que recibirá el usuario es la relativa al número CELEX del documento, el título oficial del acto y las ediciones del Diario Oficial en cuyas

Scire. 15 : 1 (en.-jun. 2009) 15-32. ISSN 1135-3716. 
lenguas el acto ha sido publicado (actualmente el DOUE se publica en 22 lenguas como regla general, y existe una edición reducida para determinados actos en irlandés), así como los idiomas en los que el acto es auténtico. En el caso del ejemplo, lo es en todas las lenguas en las que ha sido publicado. (A veces encontramos actos, como los relativos a política de la competencia, cuyos procedimientos de adopción se siguen en uno o varios idiomas, pero normalmente no en todos, y para los cuales es fundamental determinar la lengua en la que el acto hace fe).

A continuación el usuario encontrará el grupo de campos relativos a las fechas. En este caso, estamos ante un acto adoptado el 21-11-2007 que entra en vigor el primer día de 2008, según se dispone en su artículo 12, y que tiene una fecha de fin de validez indeterminada. Algunos muestran la fecha en que deben ser ejecutados por las autoridades nacionales (como las directivas). En otros casos, igualmente, se pueden dar fechas diferentes para la entrada en vigor y para la puesta en aplicación del acto. Si se establecen en el acto distintas fechas de aplicación para las diversas partes del mismo, el usuario encontrará igualmente esta información, con una remisión al artículo u otra disposición donde se determinan dichas fechas.

A continuación, se muestran tres campos con información relativa a útiles de información documental: el descriptor EUROVOC (una gran parte de las noticias

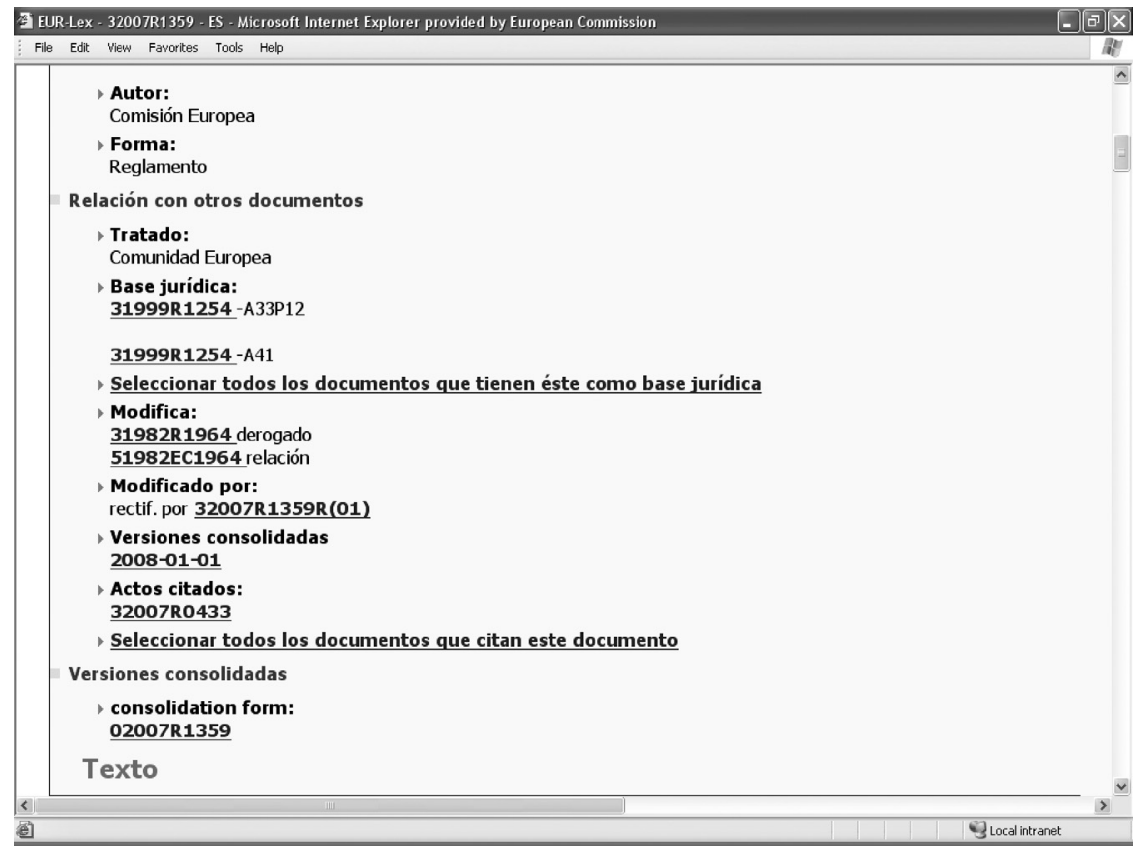

Figura 9. Noticia documental (II).

Scire. $15: 1$ (en.-jun. 2009) 15-32. ISSN 1135-3716. 
EUR-Lex son indexadas con arreglo al tesauro EUROVOC, la responsabilidad de cuya gestión recae igualmente en la Oficia de Publicaciones de las Comunidades Europeas), el código del repertorio de la legislación comunitaria vigente, y el código de materia, sistema de indexación perteneciente a la antigua base de datos CELEX.

Posteriormente, la noticia muestra la información relativa a la forma y autor del acto jurídico y después un bloque de información denominado genéricamente relación con otros documentos, donde el usuario podrá encontrar cuál es el tratado en el que se encuandra el acto jurídico y cuál es su base jurídica, con un nivel de granularidad muy desarrollado: en el presente caso, se dispone que las bases jurídicas del acto concernido es el Reglamento 1524 de 1999, concretamente sus artículos 33 (párrafo 12) y 41; inversamente, pueden recuperarse los actos que tienen como base jurídica el acto cuya noticia estamos viendo.

Siguiendo este principio de "pares de campos", se tiene acceso a las noticias de los actos afectados por el acto en cuestión (en este caso, el Reglamento 1964 de 1982, derogado por el acto de nuestro ejemplo, y el proyecto EC que se había adoptado para codificar el acto de 1982 y que, lógicamente, debe quedar relacionado con el acto derogador). Paralelamente, la noticia nos muestra los actos que tienen un rol activo respecto al de nuestro ejemplo, en este caso, un corrigendum. Los rectificativos son reconocibles porque portan el mismo número CELEX que los actos corregidos, con el añadido de la letra $\mathrm{R}$ y un número secuencial correspondiente al orden de publicación de los sucesivos corrigenda. El análisis y seguimiento de los rectificativos es especialmente complejo, ya que, normalmente y por su propia naturaleza, no obedecen a un principio sinóptico, sino que, al contrario, lo habitual es encontrarlos solo en una o varias lenguas oficiales.

Tras base jurídica / seleccionar documentos que tengan este como base jurídica y modifica / modificado por, el usuario podrá encontrar otros dos pares de campos en cada noticia documental. El primero, afecta a / relacionado con, está pensado para conectar entre sí los diferentes actos que se suceden en el procedimiento legislativo comunitario (por ejemplo, una propuesta de modificación de un reglamento plasmada en un documento COM de la Comisión, o un informe del Comité Económico y Social sobre dicha propuesta, y el reglamento cuya modificación se propone están unidos por medio de este par de campos, cuyo nombre en inglés quizá sea más clarificador de su función: earlier related instruments / subsequent related instruments). Otro par de campos que hemos de destacar es actos citados I seleccionar todos los documentos que citan este documento.

Por último, debe decirse que los documentos del sector 6 (jurisprudencia) tienen su propio sistema de relaciones entre ellos y que además estos son conectados con la legislación igualmente cuando una sentencia u otro acto jurisprudencial afecta de cualquier manera (derogándolo, anulándolo, interpretándolo, confirmando su validez, etcétera) a un acto legislativo. 
Merece ser destacada por su importancia práctica la visualización bilingüe del texto de la noticia, funcionalidad muy utilizada en los trabajos de traducción. En el caso de nuestro ejemplo, se ha elegido la opción español/polaco para el reglamento.

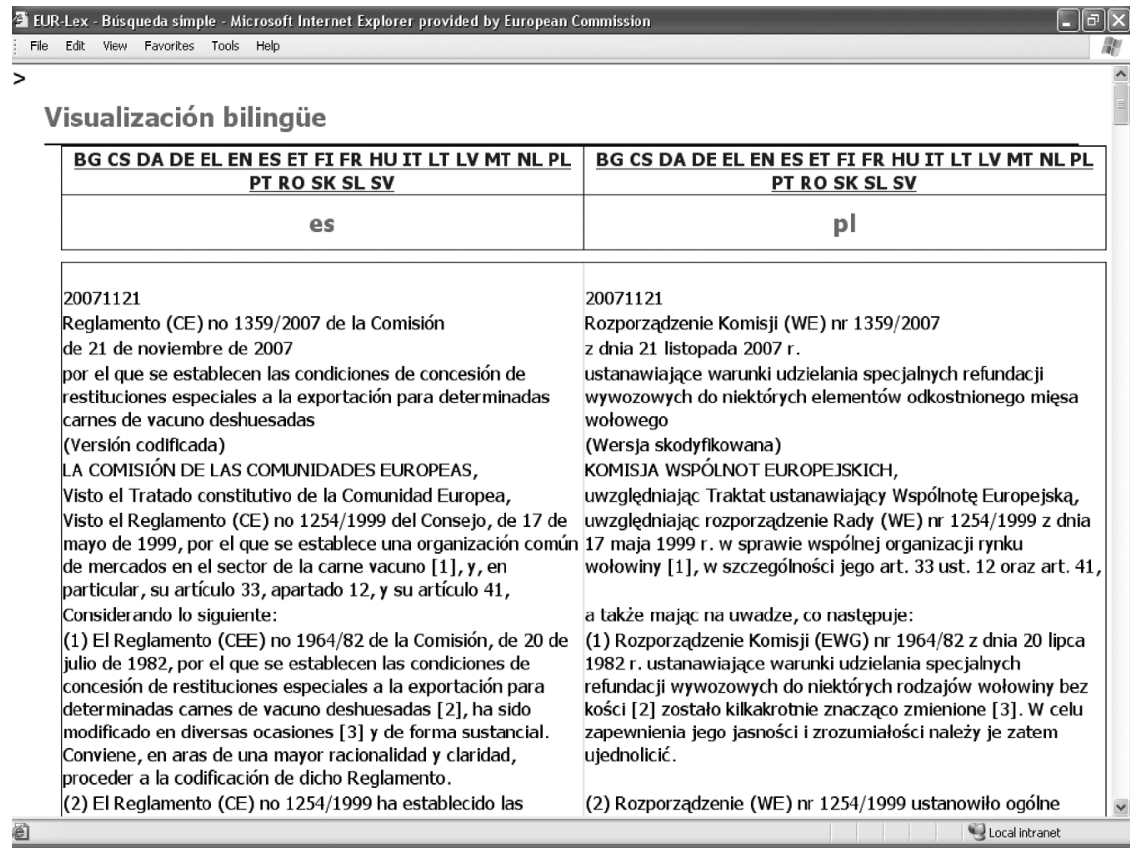

Figura 10. Visualización bilingüe del texto.

\section{Conclusiones y futuros desarrollos}

Hasta aquí hemos expuesto globalmente las características y el contenido de EUR-Lex. Hemos tratado de realizar un repaso de las fuentes del derecho de la UE y del problema real de la recuperación de la información relativa a este sector del conocimiento jurídico, no centrándonos solo en la parte de la legislación más conocida (reglamentos o directivas), sino más bien en áreas del derecho CE menos "populares", como los acuerdos internacionales, las medidas nacionales de implementación de las directivas o las disposiciones del derecho primario (tratados). Nos hemos detenido especialmente en explicar el concepto de número CELEX, el método sin duda más preciso para la recuperación de la información contenida en EUR-Lex, haciendo referencia a las distintas opciones de búsqueda y navegación

Scire. 15 : 1 (en.-jun. 2009) 15-32. ISSN 1135-3716. 
de las que dispone el usuario. Por último, hemos recorrido brevemente la noticia documental y su contenido de metadatos, auténtico valor añadido de EUR-Lex.

En cuanto al futuro, la Oficina de Publicaciones prevé, entre otras mejoras, dotar a EUR-Lex de un sistema de notificación al usuario por correo electrónico o RSS, completar las colecciones de documentos no publicados en el Diario Oficial, como los documentos COM o SEC, crear el sector 8, que recogería la jurisprudencia de los tribunales nacionales en materia de derecho comunitario, y mejorar la manera en que son mostradas las medidas nacionales de ejecución de las directivas (sector 7), con la perspectiva de, en el futuro, dar acceso no solo al derecho comunitario, sino también a los textos completos de las disposiciones nacionales que lo aplican.

\section{Documentación oficial y recursos sobre EUR-Lex}

Preguntas más frecuentes: http://eur-lex.europa.eu/es/tools/faq.htm

Guía breve sobre búsqueda simple (en inglés, francés o alemán): http://eur-lex. europa.eu/es/tools/help_miniguide.htm

Guía sobre la búsqueda avanzada (en inglés): http://eur-lex.europa.eu/en/ tools/help_advanced.pdf 